\title{
Editorial
}

\section{Improving Health of Diverse Populations}

\author{
Akiko Kamimura*, Samin Panahi and Shannon Weaver \\ University of Utah, Salt Lake City, Utah, USA
}

The issue for July 2018 mainly focuses on health of underserved populations in the United States (US), except three articles - one from Vietnam, one from the United Kingdom (UK), and the other from Poland. Three of the articles from the US - "Physical Activity Education for Adults with Refugee Background in the United States" "Targeting Diet and Physical Activity: Reaching Homeless Adults through Customized Interventions," and "Prevalence of Sarcopenia and Sarcopenic Obesity Vary with Race/Ethnicity and Advancing Age" studied lifestyle-related issues among diverse populations. Physical activity is very important to control weight and reduce the risk of developing chronic diseases such as cardiovascular disease and diabetes [1]. One of the negative health consequences from physical inactivity is obesity [2]. However, underserved populations tend to face barriers in being physically active including higher levels of stress, less access to facilities, and less time for recreation [3]. These three articles provide valuable information to promote healthy lifestyle among underserved populations in the US.

The other three articles from the US examined the issues experienced by very specific minority groups - one about African American women and lupus ("If You Don't Put it in Your Mind, then it Don't Matter: A Phenomenological Study of Coping Self-Efficacy in African American Women Diagnosed with Lupus"), one about stress management classes for uninsured free clinic patients ("Stress Management Classes for Uninsured Free Clinic Patients in the United States"), and the other about immigrant women and intimate partner violence ("Experiences of Immigrant Women Who Applied for Violence Against Women Act (VAWA) Self- Petition in the United States: Analysis of Legal Affidavits"). Since disease-related coping, stress management, and intimate partner violence (IPV) are closely tied with social circumstances, it is very important to better understand these issues to improve health and wellbeing of these populations. Culturally competent care is a key to improve coping process among African American women with lupus. For immigrant women who have experienced IPV, educational opportunities would help achieve better well-being.
Since low-income individuals such as free clinic patients face a number of stressors, stress management classes are beneficial to cope with stress. The articles will contribute to improving health and well-being of the underserved populations.

Three of the two international studies - one from Vietnam ("The Status of Baby Friendly Hospital Initiative under Hospital Quality Assessment Criteria Implementation: A Report in Vietnam") and the other from Poland ("Patient Satisfaction with Services Provided at Night and the Assessment of the Quality of Care in the Hospital") - aim at improving the quality of care. Quality of care is very important to obtain desirable health outcomes and reduce mortality [4]. These two articles will contribute to the international efforts to ensure quality of care. Finally, a study from the UK "Barriers and Enablers for UK Home Grown South Asian Prospective Students Choosing Nursing and Midwifery Courses and Careers" explored the ways to support UK born South Asians to seek nursing and midwifery careers. Promoting diversity in health care workforce is essential to reduce health disparities experienced by minority populations [5].

\section{References}

1. https://www.cdc.gov/physicalactivity/basics/pa-health/ index.htm

2. https:/www.cdc.gov/physicalactivity/community-strategies/ index.htm

3. Mendoza-Vasconez AS, Linke S, Munoz M, Pekmezi D, Anisworth C, et al. (2016) Promoting physical activity among underserved populations. Curr Sports Med Rep. 15: 290-297.

4. http://www.who.int/maternal_child_adolescent/topics/ quality-of-care/definition/en/

5. http://www.ncsl.org/documents/health/ Workforcediversity814.pdf
ADDRESS OF CORRESPONDENCE: Akiko Kamimura, $\mathrm{PhD}$, MSW, MA, Department of Sociology, University of Utah, $380 \mathrm{~S}$ 1530 E, Salt Lake City, Utah 84112, USA, Tel: +1-801-585-5496; Fax: +1-801-585-3784; E-mail akiko.kamimura@utah.edu 